\title{
How is One in Time
}

\author{
Xiaodan Liu ${ }^{1}$, Cheng Zhang ${ }^{2}$ \\ ${ }^{1}$ School of Guangdong University of Foreign Studies South China Business College, \\ ${ }^{2}$ Shaanxi Commercial School \\ ${ }^{1}$ E-mail:156612120@qq.com
}

\begin{abstract}
The phenomenological movement is a movement of continuous innovation and development of (phenomenological) thought itself. Starting from the question of "How Is One in Time", this thesis analyzes the observing ego and the pure ego in the immersive sensory state from the perspective of phenomenology. This leads to the conclusion that the observing state of the ego is the process of taking objects into the sphere under the action of knowing, and that the observing state of the ego is also the collector of elements. Finally, it is pointed out that internal time is the pure ego in the immersive state of sensation, which is also an extension of the nature of the whole without detail segmentation.
\end{abstract}

Keywords: Husserl, phenomenology, internal time

\section{INTRODUCTION}

The phenomenological movement is a movement of continuous innovation and development of (phenomenological) thought itself. A large number of representative figures emerged in the early 20 th century. Husserl left behind more than 40,000 pages of manuscripts and a large number of detailed annotations during his life, and he was a veritable "man who thought with his pen". After World War II, the new research center of phenomenology moved to the other side of the United States and evolved into various branches of phenomenology, which also used the theories and methods of realism in social science and spiritual science. The phenomenological movement is like a big tree, which Husserl is the root, Heidegger, Scheler, and others are the trunk, and the other branches are the branches and leaves. Husserl said: "Every intuition originally given is the legitimate source of knowledge, and everything that is offered to us originally in intuition (to some extent, in a living presentation) is to be accepted simply as what is presented to itself. This is only in so far as it presents itself." The pure ego and pure consciousness obtained by complete reduction without any assumption of dependence, but pure consciousness is not a mere consciousness, but a "domain" of pure consciousness, containing self-explanatory acts of consciousness such as impressions, recollections, outlooks., acquired in the original. People construct time from pure consciousness. The author will elaborate on "how is one in time".

\section{THE OBSERVING EGO AND THE PURE EGO IN THE IMMERSIVE SENSORY STATE}

What does one do in time? Time is the display of extensibility, which is a kind of negation of itself. While phenomenology prescribes cosmic time and phenomenological time, it is the presentation of two kinds of extensibility.

As the extension of the "essence of feeling" (non-intellectual) given by the phenomenon material experienced by consciousness, and the extension of objective space, the time of phenomenon is that our feeling has a beginning and an end. It is enriched by it in infinite objective time.

Phenomenological time is the way in which internal consciousness senses phenomenal materials that are themselves in cosmic objective time. They encounter each other in cosmic time and space, and continue to do so in a region that is precisely the edge of the beginning of sensory time within phenomenology. In other words, our perception is the essence of an "encounter". This "essence" comes from the a priori, from the "pure ego" behind the ego that cannot be pointed out. My perceptual experience is often temporally present in two states, which are an observing state and an immersive sensory state.

Is it true that the "I" and the world that presents the phenomena are two worlds of "seeing eye to eye"? Not 
really. At least my physical body is a part of the physical world. My physical body is always operating in the objective time and the cosmic objective time, including the observing state of me, that is, the "ego" is also concerned with the existence of the cosmic temporality. It is a kind of "orientation", and the most direct form of the external "orientation" of the "ego" is "temporality". So the "ego" is destined to be an existentialist besorgen, a source of "dasein"[3]. The "pure ego" as the "spiritual soul" sometimes enters the "sight of the ego" and sometimes does not, or alternately at intervals. Between these two times when it comes out of "sight", it is the whole time of "feeling", that is, the whole process of internal time. It is the separate existence of the "pure ego", the existence without the monitoring of the "ego", and the immersion of the "pure ego" in the phenomena. It is the objectification of the essence of the "pure ego", the transition of the "pure ego" into the phenomena, and the absence of the "ego".

\section{OBSERVING STATE OF "EGO" IS THE COLLECTOR OF ELEMENTS}

In a sense, everything runs in time. Whether it is observing the "object" or "feeling", they are all getting along with "time". What we observe is objective time (cosmic time), and what we feel is internal time, so we should first distinguish what observation and feeling refer to here. The "observation" here is a process of absorbing the phenomenon object into the category under the motivation of self-reason, and our attention is actually the category. In the experience flow, before the direct giver is synthesized, its constituent elements develop in an objective time, but there are certain forms of retention, which are held together in a certain period of time. As a whole of meaning, the "ego" is allowed to ingest the elements into the category, to deal with the transcendental patterns in the concept of pure understanding, so as to become the object of knowledge. So at this point, observation is more like a collector.

Because of the involving category and the starting point of the transcendental pattern, the observer pays special attention to the intuitive state of co-existence, the new variation, the disappearance of meaning and so on. There is a lot of coupling, which may not be kept at the same time, and some of them lose their style first, which can be called the beginning of "metamorphism". Just like when we were lying on the grass in our childhood, watching the clouds in the sky look like a horse, with the dissipating meaning of the horse, and then turn into a sheep. At this time, the mission of category, concept and transcendental pattern makes us pay special attention to the comprehensive preservation of this comprehensive phenomenon material[5]. Specifically, we pay attention to the marginal domain of the essence and form after formal synthesis, and this attention to the marginal domain of the essence of form and object is finally implemented on the scale of time. It can be said that compared to the "immersive feeling", the sight of observation (in a broad sense) focuses on the boundary between the objects or the details of the scene, because this boundary is the initial information of their respective "holding" states. It is directly related to the category, concept and qualification of a priori schema. This qualification is constantly changing with the development of time, and there are new miscellaneous concepts into the category of transcendental schemata with the old miscellaneous out of the category, conceptual transcendental pattern, which is the immediate construction and development of "meaning".

\section{INTERNAL TIME IS A KIND OF ESSENTIAL EXTENSION}

The ego is always an existence which concerns about the "meaning", even saying that the ego does not pay attention to the object, but only pays attention to "become" the meaning of the object. The ego is a supervisor, which supervises that the innate "pure ego", which has a priori category, concepts and patterns, becomes the "meaning" for the "ego" to absorb the external world norms. Therefore, the "temporality" of observation is revealed at any time and can be measured and recorded.

It is completely different from another time experience, the "feeling of immersion" experience, or even the opposite. For a feeling, such as happiness, when people change from observation to immersive existence, the ego gradually fades away. The pure ego simply faces the directness of being given, and its attention to the so-called time is only at the beginning and the end. Husserl said that the marginal domain of the experience flow, and in between, all the miscellaneous outside is presented as a whole[1]. Many changes in the boundaries between them are not within the scope of attention. For the priori, concepts and patterns of "pure ego", there is no "ego" to be involved in comprehensive planning label "miscellaneous", for its meaning, but the natural coupling into the category of transcendental patterns, and so on.

As the saying goes, the clouds come out of the forest unintentionally, and the birds fly wearily and return to the forest. Whether it enters forcefully the category, or does not force the miscellaneous to enter that, as long as it is embedded in the category, it will naturally produce "essence". The essence at this time is different from the that under observation, which is the intuitive "essence". Because the internal time as time appears only at the beginning and end of the experience, it is hidden in its process. So it is impossible to measure and record, there is no scale, even no length, only strength. When we are immersed in a fragrance of flowers, it is a "domain" with pictures, but we do not need time to describe it, but to record and remember it with intensity and depth.

Therefore, why is it called "time" if it is not presented 
in terms of length? How to reflect the continuity? In Husserl's explanation, internal time is a kind of extension and the spread of "essence". It is a kind of extension of the essence of chaos without detail segmentation as a whole, rather than the extension of changes under the spatial breadth of many contents in the form and structure. Therefore, Husserl said that the objective time of the universe is a continuous extension of space, which is not much different from Bergson's view of time and space.

At the moment when the feeling of immersion ends, the ego finds its place in cosmic time. In the immersive feeling, the recollection of the experience fragments of the past appears in the sensory period with a symbolic gesture and a non-intellectual channel, which is a "variation" of the previous phenomenon, and the "immersive feeling" enters the "internal vision". It is different from the "recollection" in the observational state, when the direct giving of reality becomes the general background of the teleprompter.

Time as an extension is always a property, and cosmic time places this fundamental property of existence in the object. Inner time, however, places this ground of being in the experiential flow of the subject. When we reflect on an experience, temporality presents itself as an object, and this presentation takes the form of sequential causal logic and quantification of length[2]. This form is actually a division and enumeration in consciousness rather than in reality. Reflection in consciousness is a spatialized presentation of temporality in non-reality, an imitation of the spatiality of reality, and an extensive treatment of extension. Why do we need to de-extend the extension? It is because that extension is a kind of substitution, a transition from one essence to another. That is, if the process is treated as an object or an object of reflection (i.e., an object of rational logic processing), the essence that has been replaced, along with the previous essence, must be listed and, as multiple links, logical cause and effect must be established between them. The very pursuit of cause and effect requires that the replacement of essence be transformed into the enumeration of essences.

This is the transition from temporal extension to spatial extension. We need to know that this is a means of reflection, precisely not a fact. "The essence of a flower is replaced by that of fruit through the stretches of time[4]." We can understand that at this point, all the materials that supported the essence of the flower are all transitioned into the essence of the fruit. In terms of material, its quantity does not change, yet the quality, as the prescriptive nature of the material, is identical with its material in real existence. Therefore, there is not a sequential enumeration in the real existence. At this point, cosmic time is more a means of reflection. For example, when a thirty-year-old John reflects on himself from the age of ten to the age of twenty to the present, in the logical process of reflection, the consciousness lists three
Johns in a sequential relationship. Obviously, the first two Johns are not real, but just virtual in process. The real extension is that temporality itself cannot be an object of consciousness. It is a thing in itself, and it accompanies the subject in the flow of experience, responding to the strength of sensation.

We are in the flow of experience, and the essence of the object exists in our consciousness as an integrated sensation. The change and replacement of the essence are also presented as a change in the strength of the senses and their replacement and are not presented in the consciousness in a sequential or quantified length; its extension is in itself. Internal time is only visible to the experiencer at the beginning and end of a sensation, so the internal time extension in itself exists in the consciousness through the mediation of the actual experience of strong and weak sensations. In turn, the temporality of observation-state reflection and that in the immersion-state experience stream are not distinctly separated in our consciousness in reality, but are intertwined. That is, we mobilize memories from time to time when we experience a scene. This memory is a kind of "essence" reproduction (virtual "enumeration") that is replaced. We cannot simply discard the "essences" that have passed, even if "they" constitute the will to believe, and the intentionality. This is the self-existence of the "cosmic temporality" in the consciousness and the flow of experience.

Cosmic time and internal time are not clearly separated, but are intermingled in the consciousness of people. One cannot leave the rational and logical reflection, nor can he or she leave the intuitive flow of experience, but a good consciousness should wander between the two without fixed dependence. Intentionality under cosmic time grasps the object with the prescriptiveness of the concept of meaning; spirituality allows us to loosen this net of meaning and grasp the strong and weak sensations of actual individuality in the experiential stream of inner time. In the back and forth of "grasping", "releasing", and "re-grasping" between cosmic time and internal time, our mind approaches the real and the profound step by step.

The harmony between the rational and the sensual is difficult for most people to achieve, because the circle of "grasping, releasing and re-grasping again" requires a consciousness that is both "loose" and "strong." This seems to be a contradiction, as reason is the judgment of the universality of concepts under logical reflection, while the immersed sensibility has to be free from the intention of this judgment of meaning. Excellent consciousness, however, has both qualities, and presents them alternately. In short, a careful balance has to be struck between the rational and the sensual. 


\section{CONCLUSION}

Continuity is not only a basic attribute of existence, but also the existence form of "ego", so the exploration of "I" is often implemented in the speculation of "continuity". The duality of ego (consciousness of master and slave) concerned by Fichte and Hegel is the existence of different nature as the basis of existence. Therefore, the "continuity" is expressed by the cosmic time in the observed state and the "internal time" in the immersive state respectively. As Bergson pointed out under the thought of spatial cosmic time and pure temporal internal time, the relationship between two kinds of continuous double ego (rational observing ego and immersion in pure ego) is also a kind of inseparable state. In this way, it provides us with space for limited relief from "limited" toil, so the excavation of this topic is of great philosophical significance to nourish the soul.

\section{REFERENCES}

[1] Husserl, Edmund. General Theory of Pure Phenomenology. [M]. The Commercial Press. 1994-03.

[2] Bergson, Henri. Time and Free will. [M]. The Commercial Press. 1987-02.

[3] Heidegger, Martin. Being and time. [M]. The Commercial Press. 2019-01.

[4] Hegel, Georg Wilhelm Friedrich. Wissenschaften der Logik. [M]. The Commercial Press. 1980-07.

[5] Husserl, Edmund. Logische Untersuchungen . [M]. The Commercial Press. 2018-04 